\title{
Kirschner Wire Migration to the Cervical Spine: A Complication of Clavicular Fixation in a Child
}

\author{
KK Tan, MS (Orth), S Ibrahim, FRCS \\ Department of Orthopaedics and Traumatology, Hospital Universiti Kebangsaan Malaysia, Kuala Lumpur, \\ Malaysia
}

\begin{abstract}
We report a case of a broken K-wire migrating to the cervical spine from the right clavicle in a 9-year-old child. The initial diagnosis, fracture of the clavicle with an acromioclavicular joint dislocation, was treated by open reduction and K-wiring. One K-wire broke and migrated to the neck, posterolateral to the $\mathrm{C} 6$ vertebra. The K-wire was removed percutaneously under image intensification. Acromioclavicular joint dislocation in children is rare since the distal clavicle does not ossify until the age of 18 or 19 years meaning that almost all closed fractures of the clavicle in children can be treated nonoperatively.
\end{abstract}

Key Words:

Fracture, Percutaneous removal, K-wire, Kirschner wire

\section{CASE REPORT}

A 9-year-old boy sustained a fracture of the acromial end of the right clavicle following a fall. The initial diagnosis was a fracture-dislocation of the right acromioclavicular joint and he was treated by open reduction and Kirschner $(\mathrm{K})$ wiring at

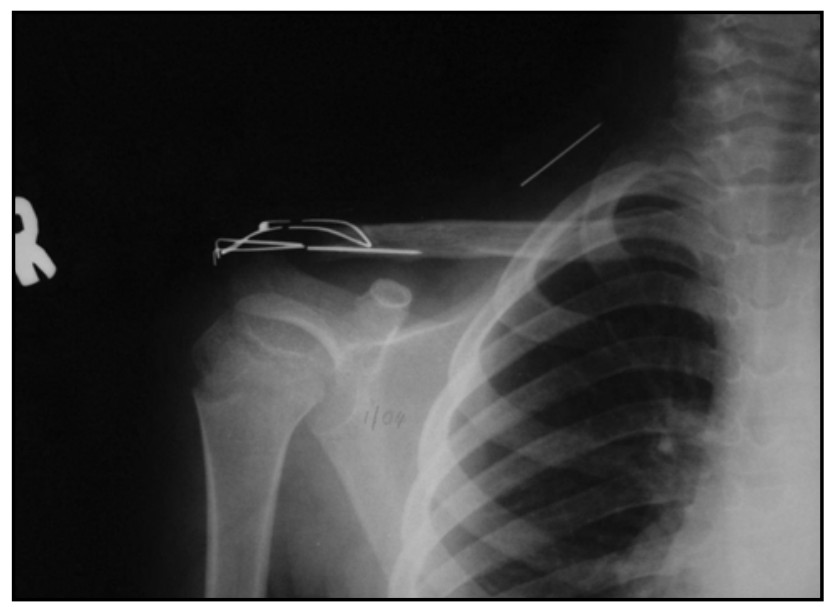

Fig. 1: Radiograph taken 9 months after injury revealed breakage of both $\mathrm{K}$-wires with one K-wire migrating into the supraclavicular region. another hospital. He was scheduled for removal of the Kwires after fracture union but did not keep that appointment. A radiograph taken 9 months after surgery showed that both $\mathrm{K}$-wires had broken and one migrated into the supraclavicular region (Fig. 1). The broken K-wires were removed by physicians at the primary hospital but the migrated K-wire could not be located. Radiography performed in August 2004 showed that the K-wire had further migrated into the neck (Fig. 2). A CT scan confirmed that the K-wire was located in the soft tissue posterolateral to the C6 vertebra (Fig 3). Although the child was asymptomatic, surgical removal was indicated as there was significant risk of the $\mathrm{K}$-wire further migrating into the spinal canal.

An open procedure through a posterior midline approach with subperiosteal dissection may result in fusion over the cervicothoracic junction. In order to prevent this complication, the K-wire was removed percutaneously (utilizing image intensification) via a small stab incision in the posterolateral aspect of the neck. The K-wire was

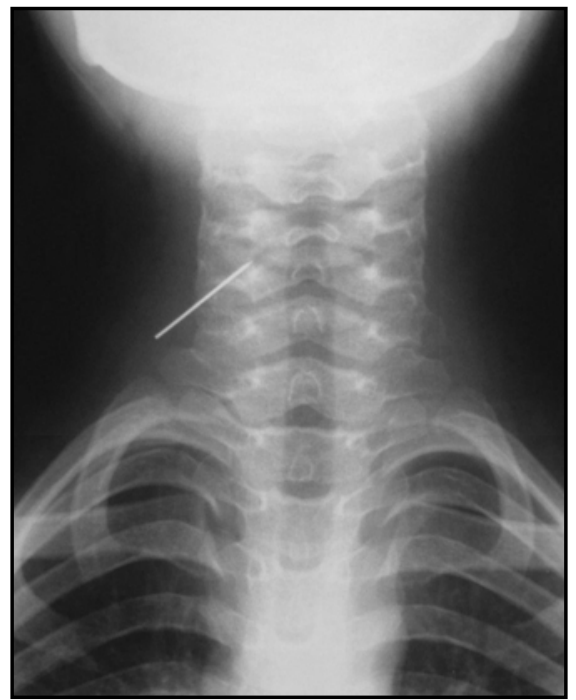

Fig. 2: Further migration of the K-wire into the neck 16 months after injury.

Corresponding Author: S Ibrahim, Department of Orthopaedics and Traumatology, Hospital Universiti Kebangsaan Malaysia, 56000 Kuala Lumpur, Malaysia 


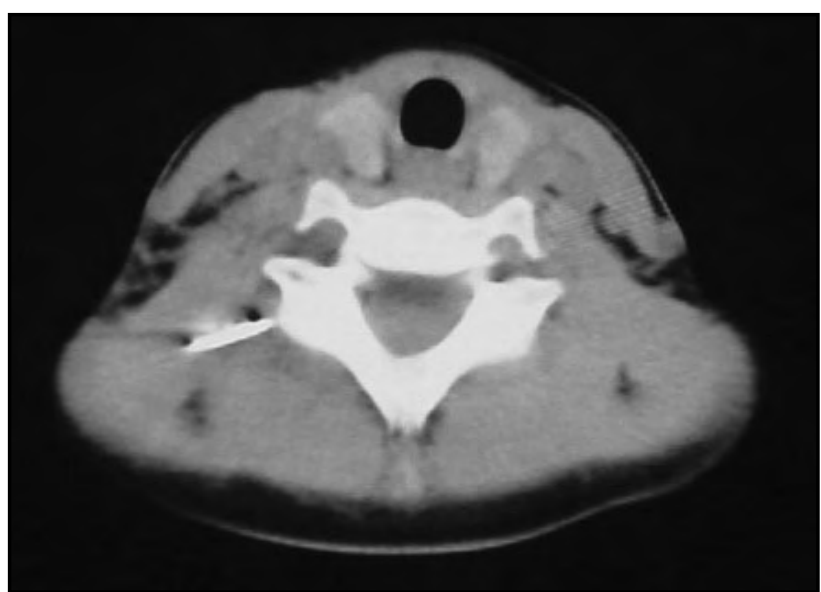

Fig. 3: CT scan showing the K-wire posterolateral to the $\mathrm{C} 6$ vertebra.

identified with a guide wire and removed with artery forceps (Fig 4). The child's recovery was uneventful.

\section{DISCUSSION}

The acromioclavicular joint and the coracoclavicular ligaments are usually intact in distal fractures of the clavicle in children, allowing for excellent potential for healing and remodelling. The distal clavicle ossifies at the age of 18 or 19 years; until that age, normal radiographic appearance may be mistaken for acromioclavicular dislocation.

There have been numerous reports in the literature of $\mathrm{K}$ wires migrating to unusual sites. Nordback \& Markulla reported migration of a K-wire from the left clavicle into the ascending aorta ${ }^{1}$. Haapaniemi \& Hermansson described an unusual complication of finger osteosynthesis in which the K-wire from the left index finger migrated to the right ventricle of the heart causing atrial fibrillation ${ }^{2}$. Leppilahti \& Jalovaara reported on 2 cases $^{3}$. In the first case, the Kwire migrated from the right clavicle to the neck, whereas in

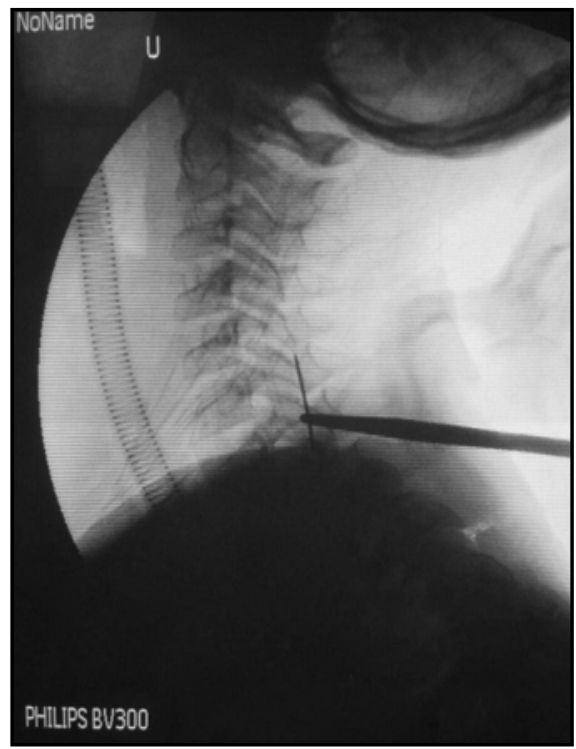

Fig. 4: Percutaneous removal of the K-wire utilizing artery forceps underguidance of image intensifier.

the second case, $2 \mathrm{~K}$-wires from the right sternoclavicular joint migrated to the mediastinum and anterior chest wall. Regel et al described an intraspinal migration of a K-wire from the right clavicle through the intervertebral foramen of C5/6 causing tetraparesis ${ }^{4}$.

The mechanism that causes or enables K-wires to migrate is unknown although muscle activity has been postulated as an underlying cause ${ }^{1-4}$. A number of fatal complications have been reported due to migration of $\mathrm{K}$ - wire into the mediastinum, heart, pulmonary artery and aorta ${ }^{3}$.

Our patient was fortunate that the K-wire did not migrate into the spinal canal. We would like to emphasise that fracture dislocation of the acromioclavicular joint in children is rare. Almost all clavicle fractures in children can be treated non-operatively.

\section{REFERENCES}

1. Nordback I, Markulla H. Migration of Kirschner pin from clavicle into ascending aorta. Acta Chir Scand 1985; 151: 177-9.

2. Haapaniemi TAT, Hermansson US. Cardiac arrhythmia caused by a Kirschner wire inside the heart: an unusual complication of finger osteosynthesis. J Hand Surg 1997; 22B(3): 402-4.

3. Leppilahti J, Jalovaara P. Migration of Kirschner wires following fixation of the clavicle-a report of 2 cases. Acta Orthop Scand 1999; 70(5): 517-26.

4. Regel JP, Pospiech J, Aalders TA, Ruchholtz S. Intraspinal migration of a Kirschner wire 3 months after clavicular fracture fixation. Neurosurg Rev 2002; 25: 110-2. 